\title{
A Survey of Systems used in the Monitoring and Control of Intravenous Infusion
}

\author{
Keerthana. $\mathrm{K}^{\# 1}$, Shree Vidhya. S ${ }^{\# 2}$, Janaki. $\mathrm{M}^{\# 3}$, Kanimozhi. J \\ \# Department of Computer Science and Engineering, \\ Sri Manakula Vinayagar Engineering College, Pondicherry, India \\ ${ }^{1}$ keerthanakaran23@gmail.com \\ 2shreevi123@gmail.com \\ ${ }^{3}$ murthy.mjjm@gmail.com \\ 4 janathakani@gmail.com
}

\begin{abstract}
Intravenous (IV) therapy is a standard form of administration of fluids and drugs to those individuals for whom oral administration cannot be done. It is used for several purposes like transfusion of blood, to treat dehydration and to fix electrolyte imbalance. However, the IV setup used in hospitals requires constant monitoring of several parameters by the clinicians such as the flow rate and level of liquid in the IV bag. A failure to do so will result in several complications which can be difficult to deal with, especially in large clinical settings. To facilitate easier monitoring of the infusion process, several systems have been developed over the years. Although the overall purpose of these systems is to monitor the flow rate and raise a warning in case of any anomaly, the techniques used to develop these systems and the functionalities fulfilled by them are subject to considerable variation. In this survey, we discuss the various systems developed to monitor the intravenous infusion process.
\end{abstract}

Keyword - Intravenous therapy, Backflow, Automatic locking, Flow Rate, IV tube

\section{INTRODUCTION}

Intravenous therapy is a form of treatment where essential fluids and drugs are passed into the body through the veins [1]. It is a routine method of infusion for individuals for ingestion of food or drugs is not possible. It is commonly used to tackle ailments leading to electrolyte imbalance, in the management of dehydration, fever, anaemia and also to administer chemotherapy [1], [2]. It is usually administered in the upper limbs due to an increased risk of thrombophlebitis during line placement in the lower limbs [3].

An intravenous (IV) setup consists of drip bottle, drip chamber, an IV tube and a roller clamp. The drip bottle is suspended from a stand at a height from the patient. The drip chamber is connected to the bottle at its' opening. The roller clamp enables the regulation of flow rate measured in drops per unit time. The infusion in the drip chamber reaches the patient as a result of the pressure difference between the drip chamber and the venous pressure [2].

Once the volume of liquid in the bottle goes below a certain level, the pressure is reversed causing backflow of blood into the capillary tube which has several adverse effects such as blockage of tube, loss of blood, swelling, infection hypothermia [4] and blood leakage [5]. Another severe effect is air embolism which is caused by emptying of the reservoir or any openings in the IV system [6] this causes an air lock in the pulmonary valve which restricts blood flow causing tissue hypoxia, reduced cardiac output and in extreme cases cause death.

Other drawbacks of IV include contamination due to microbes and backflow of patient fluids and changes in the drip rate [2], [7], [8]. Changes in the infusion rate both ways can cause adverse effects. If the patient receives less fluid than needed, it can result in dehydration and metabolic imbalance. Otherwise, if more fluid reaches the patent, it can cause hypertension, electrolyte imbalance and metabolic disturbances [2]. 


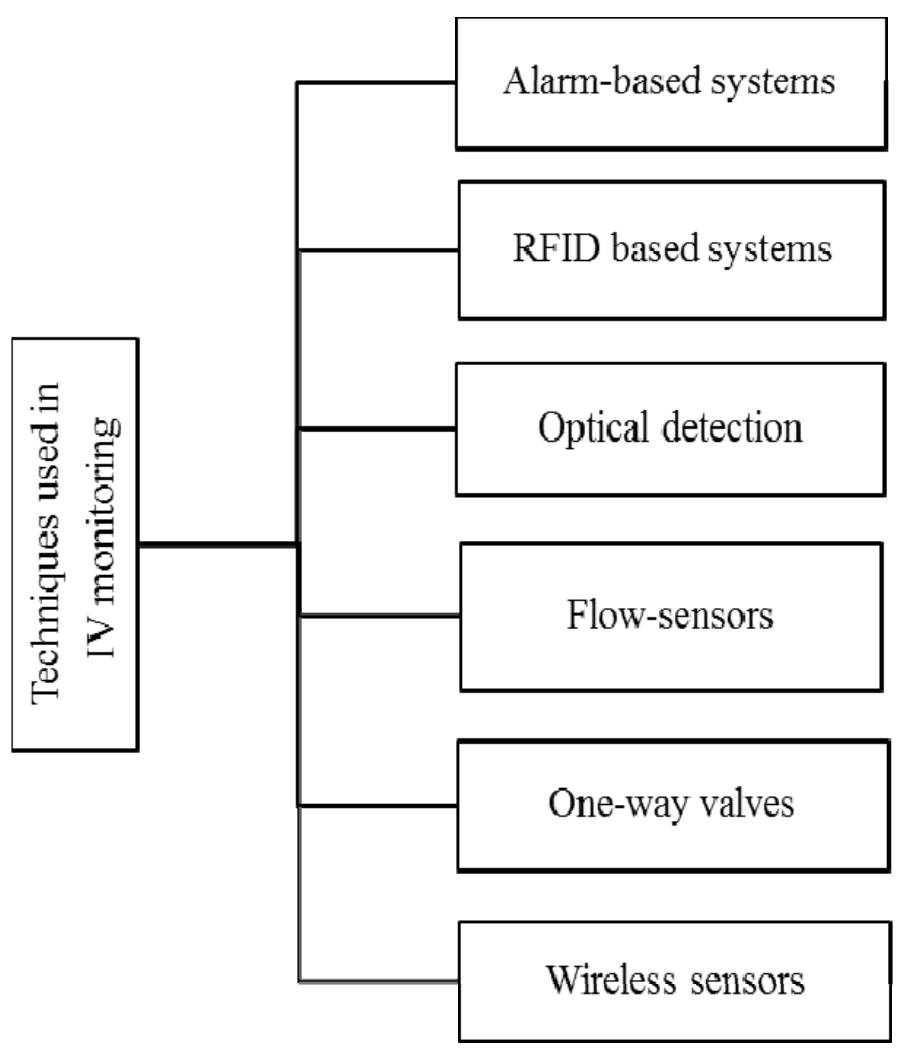

Fig.1. Techniques used in intravenous infusion monitoring systems

To prevent backflow, a method is being adapted in hospitals wherein a blood pressure cuff is tied on the same arm as the catheter is passed beneath the cuff. In case, the length of the tube is short, an extension tube is added. The cuff is inflated when necessary, causing constriction of the lumen thus preventing backflow [9]. Although this method is quite simple and has not been proven to have any adverse effects such as disconnection of the tube or leakage of fluid at the site of injection, it requires constant monitoring by clinicians. Apart from backflow, intravenous therapy also requires tremendous effort on the part of the nurses not to mention continuous surveillance of the patients' status [10] which is difficult to do in poor resource locations.

In recent years, several methods [1]-[16] have been developed to make the process of monitoring intravenous infusion easier both for the patients and clinicians. Although the overall purpose of these systems is to monitor the flow rate and raise a warning in case of any anomaly, the techniques used to develop these systems and the functionalities fulfilled by them are subject to considerable variation. The various techniques for the development of these monitoring systems is given in fig.1. In this survey, we have discussed the various techniques and functionalities of the systems used in intravenous infusion regulation.

\section{SURVEY OF SYSTEMS}

In this segment, the various control systems that have been developed to facilitate automated monitoring and flow regulation of intravenous infusion lines on in-hospital patients are discussed. A variety of techniques have been used in the implementation of these systems. The techniques include radio frequency identification, one way-valve, wireless sensors, flow rate sensors and optical detection. The purposes of these systems also vary from flowregulation to alarm based monitoring systems to notify the nursing staff in case of tube blockage or similar emergencies. In this survey, we review the systems based on the various techniques used. The various techniques are listed below:
A. Alarm based systems
B. RFID based systems
C. Optical detection
D. Flow rate sensors
E. One-way valves/non-return valves
F. Wireless sensors 


\section{A. Alarm-based systems}

Several authors have proposed an intravenous infusion monitoring and alarm-based system. Jianwen et al used a photoelectric sensor technology and signal processing system to display and monitor the velocity of the fluid and also to indicate the blockage or end of an infusion process using an alarm. C language was used to design the software. The hardware system consists of an infrared detection unit, SCM (Single chip Microcomputer) processing unit, data display module, sound and light alarm module, the locking module and wireless communication modules. The SCM and the digital display are used to count and display the level of the fluid along with the flow rate and also to raise an alarm whenever the IV bottle becomes empty. The monitoring system in general consists of two modules known as the collector and monitoring center. The collector module includes the process involved in collecting and displaying the information regarding the infusion process, to raise an alarm signal when needed. The monitoring center is useful for real-time monitoring of the patients' infusion status. This is done with the use of advanced wireless network communication wherein the data is transmitted to the monitoring center computer which is programmed to handle the data received [11].

The study conducted by Shelishiyah et al. focused on solving the blood backflow problems occurring in neonatal sections. The system consists of slotted interrupter modules which are IR sensors used to monitor the flow of fluid in an IV tube. The IR sensor used in this design was MOC 7811 which is an obstacle detector. The sensor output was tested with a CRO (Cathode Ray Oscilloscope) and a multimeter. The system incorporated an ADC0804 and AT89c2051 as the microcontroller. Since it accepts only digital signals, the analog output signals emitted by the sensor were given to an analog to digital convertor. This ADC output is then fetched by a microcontroller which is programmed to activate a voice module. The voice module used here is ISD 1820 which is a microcontroller-compatible voice recorder. This alerts the nurse on the end or blockage of an infusion process [4].

Bhavasaar et al. emphasizes intravenous liquid monitoring and alarm system using load cell as well as heart beat sensors. This method lowers the chance of heart attacks and reduces the complications in IV therapy. The main objective of this system is to monitor the level of liquid in the IV bottle and to check whenever the level drops below the set point. The level of the liquid is measured by means of a load cell which senses the weight of the IV bag. When the value recorded by the load cell falls below the set point, the buzzer arrangement alerts the medical staff appropriately. The presence of air-embolism is detected by means of a heart beat sensor which is suspended at the upper part of the catheter thus providing sufficient time for clinicians to attend the patient. The output voltage generated by the sensor is then fetched by the microcontroller thereby resulting in a voltage difference. This difference is directly proportional to the light intensity detected by the sensor, which when amplified aids in the detection of air-embolisms. [1].

Also, Raghavendra et al. designed an automated system to monitor and control the quantity of the liquid to be delivered to the patient. The design uses a light source and a light detector mounted over the drip chamber to detect the flow drops. The drip rate is computed by the microcontroller by processing the output signal received from the light detector. A user interface in the form of a keypad is also included to input parameters such as the drop factor of the IV setup, infused volume as well as the time of infusion. The input parameters processed by the microcontroller are then forwarded to the control circuit to regulate the drip rate. An alarm is also raised whenever the drip chamber is full, drip bottle is empty, pre-set value is reached, variations in drip rate or when the battery is low. Thus, the drip rate is maintained at a constant value irrespective of the variations in liquid concentration, viscosity, level of liquid in the IV bottle or other parameters. [2].

\section{B. RFID based systems}

RFID system uses tracker tag system, these tags consist of electronically stored information and the passive tag collects information from the nearby available RFID reader. In this technique the major components are load cell of s-type, drip bag weight scale, RFID/NFC tag reader, 6502 microcontroller system. The load cell transforms the tension pulled by a drip bag, to weak electrical signal. Then the electrical signal is amplified and is filled into a 16-bit A/D converter. Finally, the tension is converted into 2-byte digital weight data. The twobyte data and five-byte RFIF data are packed as a data packet which is transmitted via UDP protocol to a data collector module of IV infusion monitoring system [10]. The system assists that nurses manage all patients' IV infusion with a real-time IV infusion indication and a complete notification. The system can also be modified to be used in monitoring drug usage of elders in homes/nursing-houses for helping nurses manage elders' drug.

A procedure of designing a RFID tag by considering the material parameters of the bag of intravenous trip is described in the following text. In this work, the prototype of tag is designed based on the concept of complex conjugated match in between the chip on RFID tag and the associated antenna, and is fabricated on a PCB. Such a design offers a low-cost solution of RFID tags which are supposed to be used in a mass volume when the present monitoring system is installed in hospitals widely. [12] 
The RFID tag is designed and attached on the bag of intravenous drip. The tag is disabled when the bag is not empty because liquid contained. The bag can be of any kinds in the market and be without any electronic attachment. Sometimes LAN (Local Area Network) has been used in the process of data transmission. Active tags have local power source and it may operate hundreds of meters away from the RFID reader [12].

The NFC/RFID reader reads the tag data stuck on a drip bag. The tag data of a drip bag is used to identify a drip bag is correct for a patient or not. The system can directly enter the monitor stage of IV infusion as long as the tag data is correct, such that nurses do nothing about the system operation. In word, nurses just hang a drip bag onto the load cell and the system plays automatically [10]. It is a complete notification process that has been given to the nurses or doctors by sending the notification with the data which is recorded in the tag reader [10].

\section{Optical detection}

Here the system contains TDR method to be monitored and is stimulated through an appropriate electromagnetic signal, typically a step like voltage pulse, which is propagated through a probe, any impedance variation will cause the partial reflection of the propagating signal through a probe. Any impedance variation will cause the partial reflection of the propagating signal. The analysis of the reflection coefficient in timedomain, $\rho$, allows the retrieval of the dielectric characteristics of the material under test, as well as of its quantitative parameters, such as in the case of the level of liquid materials [13].

While considering the case of the liquid sample having a certain level, at the air-to-liquid interface an impedance chance occurs, due to the difference in dielectric permittivity. Therefore, the measured reflection coefficient shows a significant variation which may be used to individuate the air to liquid interface. Here the non-invasive probe is made of two strip electrodes, attached on an external surface of a container in which the medical liquid is contained. The ad-hoc probe configuration was realized through two adhesive copper strips with a width of $3 \mathrm{~mm}$ and a mutual distance of $3 \mathrm{~mm}$ [13].

A microwave reflectometry-based measurement method for real-time continuous monitoring of the IV drip infusion is investigated. The monitoring system presents the advantages of high accuracy, modularity, low cost, and portability that make it particularly attractive for practical in-situ medical applications [13].

The TDR measurements were performed through the HL1500 TDR unit, which generates a step-like signal with a rise time of approximately 200 PS (corresponding to a frequency content of $1.7 \mathrm{GHz}$ ). The used instrument is a low-cost portable unit which also permits the measurement multiplexing. The instrument was interfaced to a PC and controlled through a Lab View program, which also provides the possibility of automatic repetition and averaging of the measurements, the data acquisition and the estimation of Lapp. Considering the initial condition (i.e.: before starting the infusion) a portion of air is present on the top surface of the liquid solution, from the simple analysis of the TDR data, it is possible to accurately individuate the beginning and the end point of the probe. The TDR measurements were performed through the HL1500 TDR unit, which generates a step-like signal with a rise time of approximately $200 \mathrm{ps}$ (corresponding to a frequency content of $1.7 \mathrm{GHz}$ ). The used instrument is a low-cost portable unit which also permits the measurement multiplexing. Thus, this process monitoring the IV infusion rate but, it does not support to stop the backflow [13].

\section{Flow rate sensors}

The piezoresistive flow rate sensor inspired by the hair cell sensor found in the fish lateral line system has been composed and fabricated. The sensor has been bonded with a 3D printed fixture and they together have been integrated on an intravenous tube. The responses of the sensor with respect to flow rates between $100 \mathrm{~mL} / \mathrm{h}$ and $500 \mathrm{~mL} / \mathrm{h}$. The flow rate has been tested using an experimental set up and it is controlled by peristaltic pump. The sensor shows both transient phase and stable phase responses [14].

A low cost miniaturized biomimetic piezoresistive hair cell sensor inspired by the fish lateral lines hair cell sensors reported in has been modified to measure the flow rate in the IV tube. The sensor has been fabricated using micro electromechanical systems (MEMS) technology and 3D printing. The primary sensitivity testing has been done in the IV tube [14].

The lateral line system is a sensing system found in many vertebrates such as fishes and amphibians. The lateral line has been experimentally identified to play a function in fish behaviours including object and prey tracking, station holding and manoeuvring, and schooling. The lateral line system fulfils these functions by detecting the flow patterns of the surroundings, which are induced by the approaching predators or the selfmotion [14].

The lateral line system has evolved to such a mature state that some blind cavefish can swim adeptly in dark water by solely relying on the lateral line to generate hydrodynamic images of its surroundings. At the heart of the lateral line system is a tiny hair cell (micro meter in diameter) that functions as a mechano-electrical transducer. The hair cell is connected with sensory neurons at the basement. Movement in the fluid medium is detected by the displacement of the hair, which fires an action potentials series across the neurons of the innervating nerve fibre, which is relayed to the brain and processing [14]. 
The design mimics the fish lateral line system hair cell sensor. The piezoresistive sensor consists of an external auxiliary hair connected to a liquid crystal polymer (LCP) membrane bearing metallic gauge line. Mechanical displacement of the hair cell induced by the flow dragging force causes the LCP membrane to undergo mechanical strain, which induces gauge line deformation and hence a resistance change. Thus, this process regulates the flow rate using the flow rate sensor known as piezoresistive flow rate sensor that resembles fish lateral system [14].

\section{E. One-way valves/non-return valves}

One-way valves or non-return valves (NRV's) are valves that are used across several medical devices as a means to prevent backflow in intravenous infusions and as a means of preventing contamination of the patients' fluids with the infusion fluids [7], [8]. These valves are specially designed allow only a designated direction of flow (DDF) [7] and can usually withstand high levels of reverse pressure before failing [Nandy]. However, two studies [7], [8] conducted on these valves have indicated that they cannot be relied upon as a means of prevention of backflow or as infection control.

Ellger et al. hypothesized that NRV's can block the flow of fluids against DDF for low rates of infusion and play an effective role in preventing microbial, particularly bacterial contamination of the infusion fluid in proximity to the valve. A study on five different models of NRVs taking 40 units of each model from different manufacturers and they were tested for rising levels of pressure against DDF and migration of micro-organisms proximal to the valve. As an outcome of the study, 40\% of the NRVs' resulted in backflow in instance of rising pressure against DDF and 30\% of samples showed migration of micro-organisms near the valve. It was thereby concluded that NRV's are not a reliable method of prevention of backflow or contamination of fluids [7].

Another study conducted by Nandy et al. to test the levels of cross-contamination in one-way valves and their efficacy in preventing the backflow of contaminated fluids. 5 models of valves, each having a flexible diaphragm kind of mechanism but varying slightly in make. Each valve had a maximum back pressure specification which is an indicator of the amount of pressure against DDF that the valve can withstand before it fails. In each case, the maximum back pressure of each valve was far more than what is normally observed in clinical settings. The valves were tested against various different infusions of bacteriophage, bacteria or dye passed against the direction of flow from the patients' side and left for 24 hours to determine the occurrence of contamination with the infusion fluid. E-coli was the chosen as the bacterium due Leakage occurred in several models of valves against direction of flow. The conclusion was that one-way valves are not reliable for prevention of contamination in case of backflow [8].

\section{F. Wireless sensors}

Wireless sensors have been incorporated into monitoring systems for intravenous infusion monitoring. Zhang et al. [15] developed a monitoring system which involved a monitoring sensor which was incorporated at an end of the infusion tube. The sensor collects signals on the progress of the infusion. The sink node is deployed at a PC in the nurses' station from where the infusion status can be monitored. The principle of the sink nodes was found to be similar to that of the monitoring sensor. The only difference was the absence of a sensor component and a waveform adjusting circuit in the monitoring sensor. The monitor software on the PC is used to generate alarms and to process signals.

It also provides information on the medicine administered, the volume, the drop velocity. The monitoring system comprised of the following components such as ultrasonic sensors, laser sensors, and single infrared emitting diodes as well as coupled diodes. In this study, the design and implementation are based on slotcoupled infrared emitting diodes. This system incorporates a coupled infrared emitting diode after taking a number of factors such as anti-interference and power consumption into account This droplet monitoring system determined the factor affecting the weight of the droplet which was based on the viscosity of the fluid as well as the drip rate. A formula was also devised by analyzing the statistics of droplets contained per millilitre of the IV bottle under varying conditions such as velocity and viscosity [15].

Bustamante et al. [16] devised a system to detect any occlusions in the catheter or to detect when the catheter is empty and reduces the need for clinical intervention. The objective was to develop location and routing algorithms for a sensor network. A study was conducted to determine the most effective method for dripping detection which then aided them in choosing a suitable method for the sensor used in this system. These methods were divided under the following sections known as ultrasounds, piezoelectric materials, applying capacitive electrodes and other optical methods. The system consists of a sensor, a radio module for low consumption, a feeding module to give an alert in case of low battery and a microprocessor. The sensor used is an optical sensor which is used to detect the dripping of fluid in the tube. 
The sensor also consists of a button to activate the wireless configuration mode when necessary. In this mode, the sensor receives a unique ID thus preventing switch insertion problems. This sensor module consists of two stages in which one is composed of LED and a phototransistor and the other includes detection and amplification of the signal received. The microprocessor receives the signal from the sensor and determines if the infusion is dripping or not and generates signals accordingly. C program was used to program the microprocessor. A double operational amplifier is also used to stabilize the reference tension and to amplify the variations in the tension value produced by a droplet [16].

\section{CONCLUSION}

In this survey, the systems developed to monitor and regulate the process of intravenous infusion have been classified and compared in relation to the various techniques used for implementation and the functionalities offered. All of the discussed systems ultimately aim at regulating the rate of infusion and sending and an alert in case of events like drastic change in flow rate, nil flow or blockage of the tube. The systems indeed ensure that the clinician takes quick action in case of an emergency through mobile alerts, voice modules or alarms. However, these techniques have yet to be adapted in clinical settings owing to a variety of factors such as affordability, reliability and ease of implementation.

Another factor to take into consideration is the fact that while these systems effectively regulate the flow rate and provide alerts in case situations like the backflow of blood occur, the clinician in charge has to come up to the patient to rectify the issue. However, despite these setbacks, these monitoring systems facilitate better management of intravenous infusion both for the clinician and the patient and further development of these devices will no doubt exacerbate their benefits.

\section{REFERENCES}

[1] M. Bhavasaar, M. Nithya, R. Praveena, N. Bhuvaneswari and T. Kalaiselvi (2016). "Automated intravenous fluid monitoring and alerting system," in IEEE International Conference on Technological Innovations in ICT for Agriculture and Rural Development (TIAR), 2016, p.77 - 81 .

[2] B. Raghavendra, K. Vijayalakshmi, and M. Arora (2016). "Intravenous drip meter \& controller," in 8th International Conference on Communication Systems and Networks, COMSNETS, 2016, p.1-5.

[3] K. Chaudhary, L. Gupta, and R. Anand, “Avoiding iatrogenic thrombo-embolism: The "KAPLIT" technique," Scandinavian Journal of Trauma, Resuscitation and Emergency Medicine, 18, pp.1-2, 2010.

[4] R. Shelishiyah, S. Suma, and R.M.R Jacob, "A system to prevent blood backflow in intravenous infusions," IEEE International Conference on Innovations in Information, Embedded and Communication Systems, 2015, p.3-6.

[5] L. E. Seiberlich, V. Keay, S. Kallos, T. Junghans, E. Lang, and A. D. McRae, "Clinical performance of a new blood control peripheral intravenous catheter: A prospective, randomized, controlled study," International Emergency Nursing, 25, 2016 , pp.59-64.

[6] P. P Ray and N. Thapa, "A systematic review on real-time automated measurement of IV fluid level: Status and challenges," Measurement: Journal of the International Measurement Confederation, 129, pp.343-348, July 2018.

[7] B. Ellger, D. Kiski, E. Diem, I. van den Heuvel, H. Freise, H. Van Aken and A.W. Friedrich, "Non-return valves do not prevent backflow and bacterial contamination of intravenous infusions," Journal of Hospital Infection, 78, pp. 31-35, 2011.

[8] P. Nandy, M. Young, S.P. Haugen, K. Katzenmeyer-Pleuss, E.A. Gordon, S.M. Retta, and A.D. Lucas, "Evaluation of one-way valves used in medical devices for prevention of cross-contamination," American Journal of Infection Control, vol.45, pp. 793-798, 2017.

[9] P. Ambesh and S.P. Ambesh, "A simple technique to prevent reverse flow of blood from intravenous line in ipsilateral arm with noninvasive blood pressure cuff," Journal of Clinical and Diagnostic Research, vol.9, UL01,2015.

[10] F. G. Chen, J. Y. Wang, S. Chen, S. C. Tu and K. Y. Chen, "A hang-and-play intravenous infusion monitoring system," in Proceedings - 3rd International Conference on Applied Computing and Information Technology and 2nd International Conference on Computational Science and Intelligence, 2015, p. 278-281.

[11] C. Jianwen and Z. Han, "Design of intravenous infusion monitoring and alarm system based on wireless communication technology," 2011 IEEE International Conference on Mechatronics and Automation, 2011, p. 130-134.

[12] C. F. Huang, \& J. H. Lin, "A warning system based on the RFID technology for running-out of injection fluid," Proceedings of the Annual International Conference of the IEEE Engineering in Medicine and Biology Society, 2011, p. $2212-2215$.

[13] A. Cataldo, G. Cannazza, N. Giaquinto, A. Trotta, and G. Andria, "Development of a remote system for real-time control of intravenous drip infusions," IEEE International Symposium on Medical Measurements and Applications, 2011, (NA).

[14] Z. Shen, A. Kottapalli, V. Subramaniam, M. Asadnia, J. Miao, and M. Triantafyllou, "Biomimetic flow sensors for biomedical flow sensing in intravenous tubes". IEEE SENSORS, (NA), (NA), 2016.

[15] Yang Zhang, Sanfeng Zhang, Yi Ji, and Guoxin Wu, "Intravenous infusion monitoring system based on WSN," IET International Conference on Wireless Sensor Network 2010,2010, p. 38-42.

[16] P. Bustamante, U. Bilbao, G. Solas and N. Guarretxena, "A New Wireless Sensor for Intravenous Dripping Detection," 2007 International Conference on Sensor Technologies and Applications, 2007, (NA). 